\title{
Antioxidant Biomedicine: From Chemistry of Free-Radicals to Reliability of Biological Systems
}

\author{
Vitaly K Koltover* \\ Institute of Problems of Chemical Physics, Russian Academy of Sciences, Russia
}

*Corresponding author: Vitaly K Koltover, Institute of Problems of Chemical Physics, Russian Academy of Sciences, Chernogolovka, Moscow Region, 142432, Russian Federation, Email: koltover@icp.ac.ru

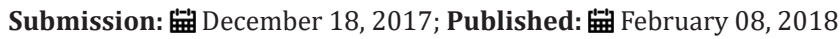

\begin{abstract}
Natural and synthetic antioxidants are widely used in modern medicine. In vitro, antioxidants effectively scavenge active free radicals thereby inhibiting free-radical chain reactions of oxidation of fatty acids, edible fats, etc. In vivo, however, neither natural antioxidants, like vitamin $\mathrm{E}$ and flavonoids, nor synthetic antioxidants, like butylated hydroxytoluene (BHT), are able to serve as efficient scavengers of oxygen free radicals. The reason is that the rate constants of the antioxidants in the reactions with oxygen free radicals and the concentrations of the antioxidants in cells and tissues are negligible as compared with the natural antioxidant enzymes to compete with the antioxidant enzymes for reactive oxygen species (ROS). Actually, the so-called antioxidants provide prophylactic maintenance against ROS by preventing the formation of oxygen radicals and free radical oxidation in cells and tissues. The protection mechanisms are different for antioxidants of different types. For example, synthetic BHT prevents generation of the oxygen anion-radical ("superoxide", $\mathrm{O}_{2} \bullet^{-}$) as the by-product of the mitochondrial electron transport, while natural flavonoids exert a preventive antioxidant action by inducing the expression of the antioxidant enzymes, superoxide dismutase and catalase. At this, the endocrine system, i.e. - prophylactic maintenance via the organism's neuro-hormonal system plays the significant role in the antioxidant therapy. Besides, there are more and more data indicating that the therapeutic effects of many pharmaceutical drugs, including antioxidants, are due to their beneficial action not only on cells and tissues of host organism but also on gastric and intestinal the organism's microbiota. Thus, true mechanisms of the antioxidant prophylaxis are to be studied on the ways of systems biology instead of free-radical chemistry. It might be said now that antioxidant biomedicine has passed a long way from "false knowledge to true ignorance".
\end{abstract}

Keywords: Antioxidants; Free radicals; Oxygen; Prophylaxis; Preventive maintenance; Biosystems; Reliability; robustness.

\section{Introduction}

The vigorous research of free radical processes and of the antioxidants in biology and medicine was stimulated by awarding the Nobel Prize in Chemistry in 1956 to Cyril Hinshelwood (University of Oxford, United Kingdom) and Nikolay Semenov (Institute of Chemical Physics of USSR Academy of Sciences, Moscow) for their studies of free radical mechanisms of chain radical reactions. By then, it was already known that free radicals, specifically the hydroxyl radical $(\mathrm{OH} \bullet)$, arise in water under the action of ionizing radiation and that toxic products of the free radical oxidation of lipids, the peroxides, appear in animal tissues on exposure to ionizing radiation (see refs. in [1,2 ]). In 1954, the idea that free radicals of oxygen are responsible for the oxygen toxicity was stated by Rebecca Gershman and Daniel Gilbert (quoted from [2]). In 1954, the signals of electron spin resonance (ESR) of the free radicals were first detected in animal tissues and in yeast cells [3]. At the same time, the free radical hypothesis of aging was put forward and inhibitors of free radical chain oxidation, antioxidants, were proposed for prolongation of life of humans and animals [4-6]. Furthermore, it was proved that antioxidant 2,6-ditertbutyl-4-methylphenol (butylated hydroxytoluene, BHT, in English literature and dibunol or ionol in Russian literature) retards the development of leucosis in mice [7]. Since then, the beneficial effects of antioxidant therapy were experimentally proved over and over again (see in refs. [8-12]).

Currently, however, the mechanisms of action of antioxidants in living systems (In vivo) do not seem as unambiguous as half a century ago. This review presents data according to which diverse physiological, in particular, therapeutic effects of synthetic and natural antioxidants are due not so much to direct radical trapping as to the ability of the antioxidants to provide preventive protection from free radicals and their chemically reactive products via the system of neuro-hormonal regulation along with the beneficial antioxidant effects on the organism's microbiota cells.

\section{Antioxidants: Setting to Free-Radical Chemistry}

By definition, antioxidants are the substances capable to terminate branching chain reactions of oxidation. The valencesaturated antioxidant molecule, containing an active hydrogen atom, reacts with an active free radical that runs the oxidative chain to give a relatively nonreactive free radical of the antioxidant molecule. The relatively nonreactive radical of the antioxidant cannot participate in chain propagation reactions and is destroyed 
upon collision with another radical (or with the vessel wall). In modern chemistry, these are mainly derivatives of secondary aromatic amines and phenols and organic phosphites and sulfides. In biomedical literature, antioxidants represent a broad class of phenolic compounds, synthetic and natural (see refs. in [8-12]). The most common antioxidants are phenolic derivatives in which the $\mathrm{OH}$ group is shielded, i.e., the so-called sterically hindered phenols. For example, in the mid- $20^{\text {th }}$ century, the synthetic antioxidant BHT found wide use as stabilizer for industrial oils and edible fats. The synthetic antioxidants based on alkyl-substituted hydroxypyridines are water-soluble, unlike BHT. With appropriate dosage, antioxidants do exert various favorable therapeutic effects. Therefore they have been long successfully used in medical practice. For example, BHT is used to treat burns, gunshot wounds, trophic ulcers, and bladder cancer. Mexidol (2-ethyl-3-hydroxy6-methylpyridine succinate) is used to treat brain circulation disorders. Emoxipine (2-ethyl-3-hydroxy-6-methylpyridine hydrochloride) is used in ophthalmology [8-12]. The same synthetic antioxidants proved to be effective geroprotectors, i.e., compounds that extend the life span of laboratory animals when added to food or drinking water on a regular basis. For example, by adding BHT to food, it was possible in some cases to extend the lives of fruit flies and of mice of some lines by $25-30 \%$. The addition of the water-soluble antioxidant, 2-ethyl-3-hydroxy-6-methylpyridine hydrochloride, to drinking water extends the average life spans of flies and mice by 24 and 38\%, respectively (see, for example, reviews $[8,12])$.

The class of natural antioxidants includes not less than twenty compounds starting from "classical" vitamin E ( $\alpha$-tocopherol) and vitamin C (ascorbic acid). Most of the natural antioxidants are substituted phenols or polyphenols, which, owing to their hydroxyl groups, are capable of inhibiting free-radical chain reactions of oxidation in model systems (In vitro), for example, oxidation of linolenic acid. Most of them are of plant origin. These are flavonoids, in particular, querticin, flavones, and resveratrol which is especially abundant in grapes and red wine, simple catechols which are present in large amounts in green tea, and catechol oligomers present in high concentrations in grapes and cocoa beans, as well as carotenoids, tannins, anthocyanins, coumarins, and hydroxycinnamic acid derivatives (see refs. in [8-12]). Currently the highest geroprotector effect has been found for resveratrol: the extension of the maximum life span by $59 \%$ in experiments on fishes and extension of the average life span by $30 \%$ in experiments on mice (on a fatty diet) [13].

It is worthy to underline, however, that the classical natural antioxidant, vitamin E, has turned out to be inefficient in analogous biomedical testing [14]. Furthermore, the results of analysis of the rate constants and actual concentrations of the antioxidants, both natural and synthetic ones, in cells and tissues also raise doubts whether the antioxidants perform In vivo their antioxidant functions in as simple way as In vitro, i.e., as the free-radical inhibitors. Indeed, chemically reactive (toxic) ROS are produced in cells and tissues of aerobic organisms. It is also known that the oxygen radical anion ("superoxide radical", $02^{--}$) is the most important source of ROS. Among the generators of 02-- in cells and tissues, there are NADPH-cytochrome-C reductase and cytochrome P-450 of endoplasmic reticulum, xanthine oxidase, catecholamine and other biogenic amines, mono- and diamine oxidases, aldehyde oxidases, oxidases of D-aminoacids, D-galactosidase, lipoxigenase, nitric oxide synthase, leukoflavines, hemoglobin and myoglobin, ascorbate, NADPH-oxidase of phagocytes and other so-called NOX enzymes (NADPH oxidases) [15]. However, the main bulk of $02^{--}$is formed as a by-product of the electron transport in mitochondria, the organelles that use up to $99 \%$ of all oxygen consumed by cells for ATP synthesis. Normal functioning of the mitochondrial electron-transport lies in the transport of electrons from the oxidation substrates, NADH and succinate, to cytochrome oxidase and, then, to oxygen with reduction of oxygen molecules to water and synthesis of ATP [15]. However, the reliability characteristics of mitochondrial nanoreactors are not perfect. As a result, normal elementary acts of electron transfers alternate with accidental malfunctions which result in formation of $\mathrm{O2}^{\bullet-}$, similarly to "recurrent failures" in engineering [9].

Besides, hydrogen peroxide $\left(\mathrm{H}_{2} \mathrm{O}_{2}\right)$ is formed as the product of the reaction of dismutation of the $02^{*-}$ radicals:

$\mathrm{O}_{2} \bullet-+\mathrm{O}_{2} \bullet-+2 \mathrm{H}+-->\mathrm{H}_{2} \mathrm{O}_{2}+\mathrm{O}_{2}$

Next, $\mathrm{O}^{\bullet-}$ reacts with $\mathrm{H}_{2} \mathrm{O}_{2}$ with formation of the $\mathrm{OH}^{\bullet}$ radical:

$\mathrm{O}_{2} \bullet-+\mathrm{H}_{2} \mathrm{O}_{2}-->\mathrm{OH}+\mathrm{OH}^{\bullet}+\mathrm{O}_{2}$

From chemistry, it is known that $\mathrm{OH}^{\bullet}$ radical is a strong oxidant which initiates free-radical reactions of oxidation of lipids and proteins, oxidative degradation of DNA with formation of 8-oxo7,8-dihydroguanine and 5,6-dihydroxy-5,6-dihydrothymine, and so on [15]. Besides, there are reasons to believe that $02^{\cdot-}$ can react with the nitric oxide (NO*) radical with formation of peroxynitrite (ONOO-), which is considered as a strong oxidant, and nitrating species that mediates biological effects of the superoxide and the nitric oxide [16].

From biochemistry, however, it is also known that there are specific enzymes, superoxide dismutases (SOD), which catalyze the dismutation of $\mathrm{O}_{2}{ }^{--}$to $\mathrm{H}_{2} \mathrm{O}_{2}$ and oxygen $[15,17]$. These enzymes, mitochondrial (Mn-SOD) and cytosol (Cu,Zn-SOD) along with periplasmatic bacterial (Fe-SOD), react with the $\mathrm{O}_{2} \bullet-$ radical anion with the rate constant $\mathrm{k} \approx 2 \cdot 10^{9} \mathrm{~L} \mathrm{~mol}^{-1} \mathrm{~s}^{-1}$ (see refs. in $[11,12$ ]. Meanwhile, the rate constants for the reactions of ascorbic acid and 5,7,8-trimethyltocol (water-soluble derivative of vitamin E) with the $\mathrm{O}_{2}{ }^{--}$radical do not exceed $10^{5} \mathrm{~L} \mathrm{~mol}^{-1} \mathrm{~s}^{-1}$, while those for hydroxypyridine antioxidants are no more than $10^{2} \mathrm{~L} \mathrm{~mol}^{-1} \mathrm{~s}^{-1}$ $[11,12]$.

Not long ago, the derivatives of vitamin $\mathrm{E}$ and ubiquinone were synthesized, Mito Vit-E and Mito $Q$, which contain, in addition to the hydrophobic antioxidant group, also a lipophilic cationic group [18]. Besides, the mitochondria-targeted plastoquinone compounds with rhodamine and triphenylphosphonium as cations were synthesized [19]. Owing to the electric charge of the cation, these molecules can use the mitochrondrial transmembrane 
potential to pass through the mitochrondrial membranes and, therefore, they get into mitochrondria, i.e., just those organelles that generate the highest amount of reactive oxygen species. The mitochondria-targeted antioxidants Mito Vit-E and Mito Q exert the beneficial effects for treating sepsis [18]. Similar antioxidants based on plastoquinone $า$ were applied profitably against the age pathologies in animals, in particular, for treating a number of cardiovascular and ocular diseases and even as geroprotectors in experiments on mice or other animal species [19]. For Mito Q (ubiquinone-based antioxidant), the rate constant for the reaction with $02^{--}$in water can be as high as $10^{8} \mathrm{~L} \mathrm{~mol}^{-1} \mathrm{~s}^{-1}$ according to pulse radiolysis data. However, in this case, too, it remains an order of magnitude lower than for SOD. In principle, mitochondria-targeted antioxidants can be accumulated in mitochondria. However, they can hardly be accumulated up to a concentration comparable with the amount of SOD (about $10^{-5} \mathrm{~mol} \mathrm{~L}^{-1}$ ) without considerable disturbance of the operation of mitochondrial nanoreactors.

Of course, inasmuch as reliability ("robustness") of the SOD protection is limited, there exists a finite probability that the 02-- radical still penetrates the SOD-barrier and then reacts with $\mathrm{H} 2 \mathrm{O} 2$ to give the hydroxyl radical $\left(\mathrm{OH}^{\circ}\right)$ which is known as the strong oxidant in chemistry. However, there are other specialized antioxidant enzymes, catalase and glutathione peroxidase, which catalyze hydrogen peroxide decomposition to water and oxygen [15]. The rate constant for the reaction of vitamin $\mathrm{E}$ with $\mathrm{OH}^{*}$ radical can be as high as $8-10^{10} \mathrm{~L} \mathrm{~mol}^{-1} \mathrm{~s}^{-1}$. Meanwhile, the $\mathrm{OH}^{\cdot}$ radical is known to react with any organic molecules as a strong oxidant with the rate constants close to the diffusion limit, $>10^{10}$ $10^{11} \mathrm{~L} \mathrm{~mol}^{-1} \mathrm{~s}^{-1}$ (see refs. in $[11,12]$ ). Therefore, In vivo, none of the antioxidants can compete for the hydroxyl radical with other organic molecules which are obviously always present around this radical in considerably greater numbers than the molecules of any antioxidants. It is known from chemistry, that peroxyl radicals $\mathrm{RO}_{2} \bullet$ can appear in reactions of $\mathrm{OH}^{\bullet}$ radicals with lipids, for example, with mitochondrial cardiolipin. The rate constants for the reactions of synthetic and natural antioxidants with $\mathrm{RO}_{2} \bullet$ in model reactions may range up to about $10^{6} \mathrm{~L} \mathrm{~mol}^{-1} \mathrm{~s}^{-1}$. In vivo, however, $\mathrm{RO}_{2} \bullet$ and other products of peroxidation arise mainly as secondary products in the reactions that accompany cell death on apoptosis and auto phagocytosis during utilization of the cellular waste by lyzosomes and peroxysomes. One can suggest that antioxidants are unlikely to be highly necessary for scavenging the active radicals in catabolism. The reports on In vivo yields of the DNA oxidation products for both mitochondrial and nuclear DNA are also overestimated due to various artifacts [20]. Thus, manifold beneficial effects of antioxidants In vivo can hardly be interpreted on the basis of simple chemical analogy with the action of the same antioxidants as radical scavengers In vitro.

\section{Antioxidants: Setting to Bioreliability}

From the general theory of reliability, it is well known that the most efficient way to increase the reliability (robustness) of any complex system is prophylaxis, i.e. - the well-timed prevention of malfunctions (failures) of functional elements from which the system is constructed (see, for example, [21]). Following this reliability-theory guide-line, a special series of experiments was performed by our group, in which it has been shown that antioxidants do provide the preventive protection from free radicals In vivo. At this, the particular protection mechanisms are different for antioxidants of different types.

For BHT, this antioxidant prevents generation of the $\mathrm{O}_{2}{ }^{--}$radicals as by-products of the mitochondrial electron transport. In the studies of low-temperature ESR (electron spin resonance) signals of rat tissues, it was found that BHT increases the myocardium oxygenation [22]. Meanwhile, hypoxia results in structural damage of mitochondrial membranes resulting in considerable decrease in the reliability of electron transport, so that the mitochondria become generators of intense fluxes of the 02*- radicals [23]. It stands to reason that BHT prevents the development of hypoxia by increasing the degree of myocardium oxygenation, thereby preventing the transformation of the electron-transport nanoreactors of the myocardial mitochondria into the intensive generators of $\mathrm{O}_{2}{ }^{--}$. Furthermore, it has been proved that BHT produces the dramatic hormonal changes in the animal's blood, i.e., the increase of corticotropin and corticosteroids along with the decrease of thyrotropin and triiodothyronine in blood plasma of rats after the BHT administration [24,25]. It means that BHT induces the substantial shift in the activity of adenohypophysis gland which is the source of corticotropin and thyrotropin hormones, and this is accompanied with the relevant shifts in the activity of peripheral endocrine glands, the adrenal cortex (the source of corticosteroids) and the thyroid gland (the source of triiodothyronine). It is common knowledge that the release of corticotropin into blood, followed by increase in synthesis of corticosteroids and decrease in synthesis of thyroid hormones, is a significant phase of the systems adaptation to stress. It seems that, with regular introduction into animals' food, BHT as a mild stress (hormetic) factor trains the neuro-hormonal system and, thus, increases the systems reliability, i.e. -adaptive capabilities of the organism [25]. Hence, BHT is actually able to decrease the level of active oxygen species in myocardial cells and, probably, other cells too. However, the beneficial effect of this antioxidant is manifested not through direct elimination (scavenge) of free radicals but in the preventive manner, i.e., upon decrease in the probability of generation of the radicals.

Another example of synthetic antioxidant is 2-ethyl-3hydroxy-6-methylpyridine hydrochloride (Emoxipine). This water soluble antioxidant was first introduced in biomedicine as the anti-radiation protector [26]. The mechanism of the antioxidant prophylaxis in this case differs from that for BHT. The antioxidants based on hydroxypyridines are analogs of pyridoxine and pyridoxal phosphate which are the group $\mathrm{B}_{6}$ vitamins. Meanwhile, pyridoxal phosphate performs the cofactor functions for glutamate aspartate aminotransferase, RNA polymerase and some other enzymes of biosynthesis of the nitrogen-containing compounds [15]. This implies that hydroxypyridine antioxidants are the anti-metabolites of vitamin $\mathrm{B}_{6}$ and, as such, they inhibit the key enzymes of synthesis of amino acids and nucleotides. In particular, this allows understanding of the efficiency of 2-ethyl-3-hydroxy- 
6-methylpyridine as the anti-radiation protector. Inhibition of biosynthesis retards cell division and, thus, provides the cells with additional time for restoring the genetic structures damaged by ionizing radiation [26].

The so-called mitochondria-targeted antioxidants, Mito Vit-E (vitamin E derivative [18]) and Sk Q (ubiquinone derivative [19]), can also act in a preventive manner. As the phenolic compounds, Mito Vit-E and Sk Q have weakly acidic properties and, as such, they can serve as protonophore un couplers of oxidative phosphorylation, i.e., they can uncouple electron transport and ATP synthesis in mitochondria like, for example, 2,4-dinitrophenol. Besides, as the hydrophobic cations, they can transfer counterions through the mitochondrial lipid membrane and, thereby, they can decrease the transmembrane potential. Meanwhile, it is generally known that the electron transport in mitochondria experiences a "back pressure" of the transmembrane potential [15]. Therefore, as the transmembrane transfer agents of protons and anions, Mito Vit-E and Sk Q decrease the transmembrane potential thereby decreasing the generation of $02^{\cdot-}$ and its active products in mitochondria. This provides grounds for believing that mitochondria-targeted antioxidants not so much scavenge directly the $\mathrm{O}^{--}$(or its protonated form $\mathrm{HO}_{2} \bullet$ ) but prevent the formation of these radicals in mitochondria $[11,12]$.

Mechanisms of antioxidant activity of natural antioxidants are being revised too. For example, glutathione (GSH), the physiological antioxidant and second messenger in cells, is considered now as the post-translational modifier that control over epigenetic mechanisms at different levels, i.e., substrate availability and enzymatic activity for DNA methylation, changes in the expression of micro RNAs, and participation in the histone code [27]. Furthermore, there are a wide class of natural antioxidants, called flavonoids, among them - querticin, flavones and resveratrol. The last one is especially abundant in grapes and red wine [13]. These antioxidants can provide the preventive protection against oxygen radicals by induction of specific antioxidant enzymes. Indeed, the induction of synthesis of SOD and catalase was detected in blood erythrocytes of humans who received the food additive Protandim (extracts from five medical plants) [28]. The authors concluded that modest induction of the antioxidant enzymes, SOD and catalase, may be a much more effective approach to the problem of defense from free radicals than supplementation with antioxidants "that can, at best, stoichio metrically scavenge a very small fraction of total oxidant production" [28]. Noteworthy, that resveratrol activates expression of the sirtuin proteins thereby providing, in part, the increase in expression of mitochondrial SOD In vivo (see refs. in [29]).

Inasmuch as expression of SOD and other antioxidant enzymes in humans and animals is under the hormonal control, flavonoids also seem to make their preventive maintenance defense through hormonal regulation mechanisms. Indeed, in the experiments with Macaca mulatta monkeys it was found that the diurnal changes (circadian rhythms) in the SOD activity in erythrocytes tightly and positively correlate with the diurnal changes in the levels of cortisol and dehydroepiandrosterone sulfate (DHEAS) in blood plasma
[30]. For young animals the values of correlation coefficient were $0.92+0.09$ (cortisol versus SOD) and $0.99+0.02$ (DHEAS versus SOD). With aging, the circadian rhythms of SOD, cortisol and DHEAS are smoothed out although the correlation between the diurnal changes in cortisol and in SOD still maintains even for old animals. These results, like the above-mentioned experiments with BHT, testify that corticosteroid hormones do play the essential role in the regulation of SOD activity.

Quite a lot of data have now been accumulated demonstrating that even vitamin E ( $\alpha$-tocopherol), a "key antioxidant", can hardly serve as a free radical inhibitor In vivo and, hence, this issue in handbooks should be revised too [31,32]. There are four tocopherol isomers: $\alpha-, \beta-$, 0 -, and $\delta$-tocopherol. All four isomers react in vitro with the $\mathrm{RO}_{2} \cdot$ radical with approximately the same rate constants, of about $106 \mathrm{~L} \mathrm{~mol}^{-1} \mathrm{~s}^{-1}$. In the living nature, however, mainly $\alpha$-tocopherol is encountered. As shown in experiments on cell cultures and isolated enzymes, the vitamin E inhibits a key regulator enzyme of biosynthesis, protein kinase C. Besides, it inhibits 5-lipoxygenase and phospholipase $A_{2}$ and activates protein phosphatase $2 \mathrm{~A}$ and diacylglycerol kinase. It was proved that $\alpha$-tocopherol modulates the expression of genes encoding synthesis of a number of protective proteins including $\alpha$-TTP, $\alpha$-tropomyosin, and collagenase. Moreover, $\alpha$-tocophenyl phosphate, rather than the antioxidant phenolic form of vitamin $\mathrm{E}$, serves as the bioregulator. It has been suggested that $\alpha$-tocopherol acts as a ligand for yet unidentified specific proteins, membrane receptors or transcription factors, capable of regulating signal transduction and gene expression $[31,32]$.

Furthermore, there are more and more data indicating that therapeutic effects of many pharmaceutical drugs are due to their beneficial action not only on the cells and tissues of the host organism but also on gastric and intestinal microbiota. Most of flavonoids, which are traditionally regarded as natural antioxidants, refer to the extensive class of physiologically active compounds long known as phytoalexins. Moreover, the phytoalexins are synthesized in plant tissues just for fighting, like antibiotics, against bacterial and fungal infections and for acting as inhibitors of transcription and translation of particular proteins in the cells of the infecting organisms [33]. Meanwhile, the number of microbiota cells in the gastrointestinal tract, on the skin and in some other organs and tissues nearly exceeds the number of cells of the host organism. Of even greater importance is that the microbial cells produce physiologically active substances that markedly affect all organs and tissues including immune system. More and more increasing number of experimental data are coming that the microbial metabolites promote metabolic benefits in the brain cells via gutbrain neural circuits (see refs. in $[11,12,34,35]$ ). As a matter of fact, a new synthetic biomedical concept has being emerged that the human microbiota is a source of therapeutic drug targets.

In view of the advances in systems biology, one can suggest that the so-called antioxidants, both natural and synthetic ones, attack the organism's microbial population. In high doses, these substances are toxic, as implied, because of their deleterious effects 
on the microbiota. In low doses, however, the same compounds produce favorable effects on the organism's microbiota, in a hormetic-like fashion, and, thereby, increase the system reliability and lifespan of the organism. One can further assume that the socalled mitochondria-targeted compounds like Mito Vit-E and Sk Q actually affect the microbiotic cells. Thus, in this century, which is the century of systems biology, the theory which was put forward in the early 20th century by Metchnikoff about the considerable effects of the microbial population on the body health and aging [36] is actually revived. One can say with reasonable confidence that "Metchnikoff arises".

It has rather long been questioned whether the synthetic and natural antioxidants work in vivo in the same way, as In vitro, i.e. - as simple chemical scavengers of $\mathrm{OH}^{\bullet}$ and other active radicals [10-12,22,24-26]. Indeed, SOD was discovered by J M Mc Cord and I Fridovich long ago, in 1968 (see refs. in [15,17]). Shortly thereafter, in the chemical kinetics experiments it was found that neither natural nor synthetic antioxidants are able to compete for the reactive oxygen species with the specialized anti-oxidative enzyme systems of cells and tissues. Over the years, more and more experimental results indicate that the true mechanisms of the antioxidant effects are to be studied on the ways of systems biology instead of freeradical chemistry. Last years, such terms as "polyphenols", instead of antioxidants, and "redox regulation/redox signaling pathways", instead of oxidative stress, came into use, at last [37]. Moreover, the Society for Free Radical Biology and Medicine has been recently renamed in the Society for Redox Biology and Medicine. Yet, the paradigm that the antioxidants directly intercept free radicals in vivo in the same manner as in vitro had a very long-lived existence. As said in [38], "it is harder to overcome old ideas, rather than create the new ones". In other words, "never attribute to malice that which is adequately explained by stupidity" (Hanlon's Razor).

\section{Conclusion}

Natural and artificial antioxidants, or polyphenols, effectively protect living cells and tissues from chemically reactive oxygen species. However, the efficiency of the antioxidants as scavengers of free radicals in living systems is low, compared to the natural antioxidant enzymes, since the rate constants and concentrations of the antioxidants in cells and tissues are negligibly small to compete with the antioxidant enzymes for the oxygen radical anions $\left(\mathrm{O}^{\circ-}\right)$ and other reactive oxygen species. In vivo, the antioxidants do decrease concentrations of the reactive oxygen species but they do it not as inhibitors of the free radical reactions but in the preventive manner, i.e., as physiologically active compounds that prevent the formation of the oxygen radicals. Actually, the antioxidants provide prophylactic maintenance against the reactive oxygen species. At this, the protection mechanisms are different for antioxidants of different types. For example, the synthetic antioxidant BHT prevents the formation of the oxygen radical anions as the by-products of mitochondrial electron transport while natural flavonoids exert the preventive antioxidant action through the induction of synthesis of the antioxidant enzymes, superoxide dismutase and catalase. The prophylactic antioxidant maintenance realizes via the organism's neuro-hormonal (endocrine) system. The antioxidant injection, as a mild stress factor, trains the neuro-hormonal system in a hormetic-like fashion and, thus, increases adaptive capabilities, i.e. - the systems reliability of the organism. Besides, there are more and more data indicating that the therapeutic effects of many pharmaceutical drugs, including antioxidants, are due to their beneficial action not only on the cells and tissues of the host organism but also on the gastric and intestinal microbiota. Thus, true mechanisms of the antioxidant prophylaxis are to be studied on the ways of systems biology instead of free-radical chemistry. It might be said now that antioxidant biomedicine has passed a long way from "false knowledge to true ignorance".

\section{Acknowledgment}

This work was realized in accordance with the current scientific plans authorized by the Federal Agency for Scientific Organizations (FASO Russia), project no. 0120-1361-868.

\section{References}

1. Bacq ZM, Alexander P (1961) Fundamentals of Radiobiology. Pergamon Press, New York, USA, p. 386.

2. Gilbert DL (1981) Oxygen and Living Processes: An Interdisciplinary Approach. In: Gilbert DL [Ed.] Springer-Verlag, New York, USA, p. 401.

3. Commoner B, Townsend J, Pake GE (1954) Free radicals in biological materials. Nature 174: 689-691.

4. Harman D (1956) Aging: A theory based on free radicals and radiation chemistry. J Gerontol 11(3): 298-300.

5. Harman D (1957) Prolongation of the normal life span by radiation protection chemicals. J Gerontol 12(3): 257-263.

6. Harman D (1992) Free radical theory of aging: History EXS 62: 1-10.

7. Emanuel' NM, Lipchina LP (1958) Leucosis in mice and some features of its development under the action of certain inhibitors of oxidizing chain processes. Dokl Akad Nauk SSSR 121: 141-144.

8. Obukhova LK, Emanuel NM (1983) Role of free radical chain reactions of oxidation in molecular mechanisms of aging of living organisms. Russian Advances in Chemistry 52(3): 353-372.

9. Koltover VK (1992) Free radical theory of aging: History. EXS 62: 11-19.

10. Koltover VK (2009) Bioantioxidants: The systems reliability standpoint. Toxicol Ind Health 25(4-5): 295-299.

11. Koltover VK (2010) Antioxidant biomedicine: from free radical chemistry to systems biology mechanisms. Russian Chem Bull 59(1): 37-42.

12. Koltover VK (2017) Antioxidant therapy of aging: from free radical chemistry to systems theory of reliability. In: Vaiserman A [Ed.] AntiAging Drugs: from Basic Research to Clinical Practice, Royal Society of Chemistry Publishing, Cambridge, UK, pp. 183-204

13. Baur JA, Sinclair DA (2006) Therapeutic potential of resveratrol: the In vivo evidence. Nature Rev Drug Discovery 5(6): 493-506.

14. Driver C, Georgeou A (2003) Variable effects of vitamin E on Drosophila longevity. Biogerontol 4(2): 91-95.

15. Nelson DL, Cox MM (2008) Lehninger Principles of Biochemistry. In: $\left(5^{\text {th }}\right.$ edn), WH Freeman, New York, USA, p. 520

16. Murad F, Barber R (2009) A hypothesis about cellular signaling with nitric oxide in the earliest life forms in evolution. Free Radic Biol Med 47(9): 1325-1327.

17. McCord JM, Fridovich I (2014) Superoxide dismutases: you've come a long way, baby. Antioxid Redox Signal 20(10): 1548-1549. 
18. Murphy MP (2008) Targeting lipophilic cations to mitochondria Biochim Biophys Acta 1777(7-8): 1028-1031.

19. Skulachev VP (2012) Mitochondria-targeted antioxidants as promising drugs for treatment of age-related brain diseases. J Alzheimers Dis 28(2): 283-289.

20. Cadet AF, Douki T, Ravanat JL (2008) Oxidatively Generated Damage to the Guanine Moiety of DNA: Mechanistic Aspects and Formation in Cells. Accounts Chem Res 41(8): 1075-1083.

21. Bazovsky I (1961) Reliability. Theory and Practice. Bazovsky Igor. Prentice-Hall, London, pp. 292.

22. Koltover VK, Gorban EN, Maior PS (1984) Mechanism of prolongation of life by dibunol (butylated hydroxytoluene). Dokl Biophys 277(2): 130133.

23. Nohl H, Koltover V, Stolze K (1993) Ischemia/reperfusion impairs mitochondrial energy conservation and triggers $\mathrm{O}_{2}{ }^{--}$release as a byproduct of respiration. Free Radical Res Com 18(3): 127-137.

24. Frol'kis VV, Gorban EN, Kol'tover VK (1985) Effect of the antioxidant butylated hydroxytoluene (dibunol) on hormonal regulation in rats of various ages. Dokl Biophys 284: 210-213.

25. Frolkis VV, Gorban EN, Koltover VK (1990) Effects of antioxidant butylated hydroxytoluene (BHT) on hormonal regulation and ESR signals in adult and old rats. AGE 13(1): 5-8.

26. Koltover VK, Kutlakhmedov YA, Afanaseva EL (1980) Recovery of cells from radiation-induced damages in the presence of antioxidants and the reliability of biological systems. Doklady Biophysics 254(3): 159-161.

27. García-Giménez JL, Romá-Mateo C, Pérez-Machado G, Peiró-Chova L, Pallardó FV, et al. (2017) Role of glutathione in the regulation of epigenetic mechanisms in disease. Free Radic Biol Med 112: 36-48.
28. Nelson SK, Bose GK, Grunwald PM, Myhill P, McCord JM, et al. (2006) The induction of human superoxide dismutase and catalase In vivo: A undamentally new approach to antioxidant therapy. Free Radic Biol Med 40(2): 341-347.

29. Houtkooper RH, Pirinen E, Auwerx J (2012) Sirtuins as regulators of metabolism and health span. Nat Rev Mol Cell Biol 13(4): 225-238.

30. Goncharova ND, Shmaliy AV, Bogatyrenko TN, Koltover VK (2006) Correlation between the activity of antioxidant enzymes and circadian rhythms of corticosteroids in Macaca mulatta monkeys of different age. Exp Gerontol 41(8): 778-783.

31. Azzi A, Meydani SN, Meydani M, Zingg JM (2016) The rise, the fall and the renaissance of vitamin E. Arch Biochem Biophys 595: 100-108.

32. Galli F, Azzi A, Birringerc M, Cook-Mills JM, Eggersdorfer M, et al. (2017) Vitamin E: Emerging aspects and new directions. Free Radic Biol Med 102: $16-36$

33. Bailey JA, Mansfield JW (1982) Phytoalexins. New York, USA, p. 334.

34. Heintz C, Mair W (2014) You are what you host: microbiome modulation of the aging process. Cell 156(3): 408-411.

35. Espin JC, González-Sarrías A, Tomás-Barberán FA (2017) The gut microbiota: A key factor in the therapeutic effects of (poly) phenols. Biochem Pharmacol 139: 82-93.

36. Metchnikoff E (1907) The Prolongation of Life. Optimistic Studies. W Heinemann, London, pp. 343.

37. Forman HJ, Davies KJA, Ursini F (2014) How do nutritional antioxidants really work: Nucleophilic tone and para-hormesis versus free radical scavenging In vivo. Free Radic Biol Med 66: 24-35.

38. Keynes JM (2007) The General Theory of Employment, Interest and Money. Palgrave Macmillan, Ney York, USA, pp. 428.

\section{Your subsequent submission with Crimson Publishers will attain the below benefits}

- High-level peer review and editorial services

- Freely accessible online immediately upon publication

- Authors retain the copyright to their work

- Licensing it under a Creative Commons license

- Visibility through different online platforms

- Global attainment for your research

- Article availability in different formats (Pdf, E-pub, Full Text)

- Endless customer service

- Reasonable Membership services

- Reprints availability upon request

- One step article tracking system 